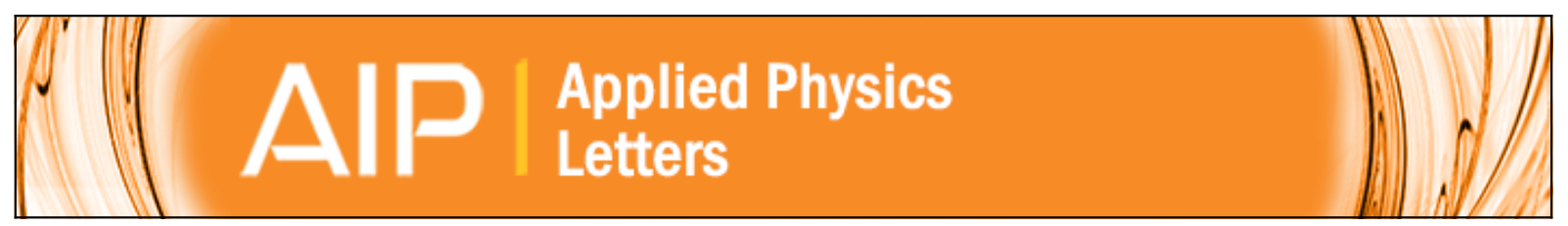

\title{
Exciton and multiexciton optical properties of single InAs/GaAs site-controlled quantum dots
}

J. Canet-Ferrer, G. Munoz-Matutano, J. Herranz, D. Rivas, B. Alen, Y. Gonzalez, D. Fuster, L. Gonzalez, and J. Martínez-Pastor

Citation: Applied Physics Letters 103, 183112 (2013); doi: 10.1063/1.4828352

View online: http://dx.doi.org/10.1063/1.4828352

View Table of Contents: http://scitation.aip.org/content/aip/journal/apl/103/18?ver=pdfcov

Published by the AIP Publishing

\section{Articles you may be interested in}

Charge state control in single InAs/GaAs quantum dots by external electric and magnetic fields Appl. Phys. Lett. 105, 041109 (2014); 10.1063/1.4891828

Site-controlled formation of InAs/GaAs quantum-dot-in-nanowires for single photon emitters Appl. Phys. Lett. 100, 263101 (2012); 10.1063/1.4731208

Photoluminescence of self-assembled InAs/GaAs quantum dots excited by ultraintensive femtosecond laser J. Appl. Phys. 106, 103522 (2009); 10.1063/1.3264624

The influence of size distribution on the luminescence decay from excited states of InAs/GaAs self-assembled quantum dots

J. Appl. Phys. 105, 033507 (2009); 10.1063/1.3073934

Effects of indium segregation on optical properties of nitrogen-doped InAs/GaAs quantum dots J. Appl. Phys. 104, 103532 (2008); 10.1063/1.3021054

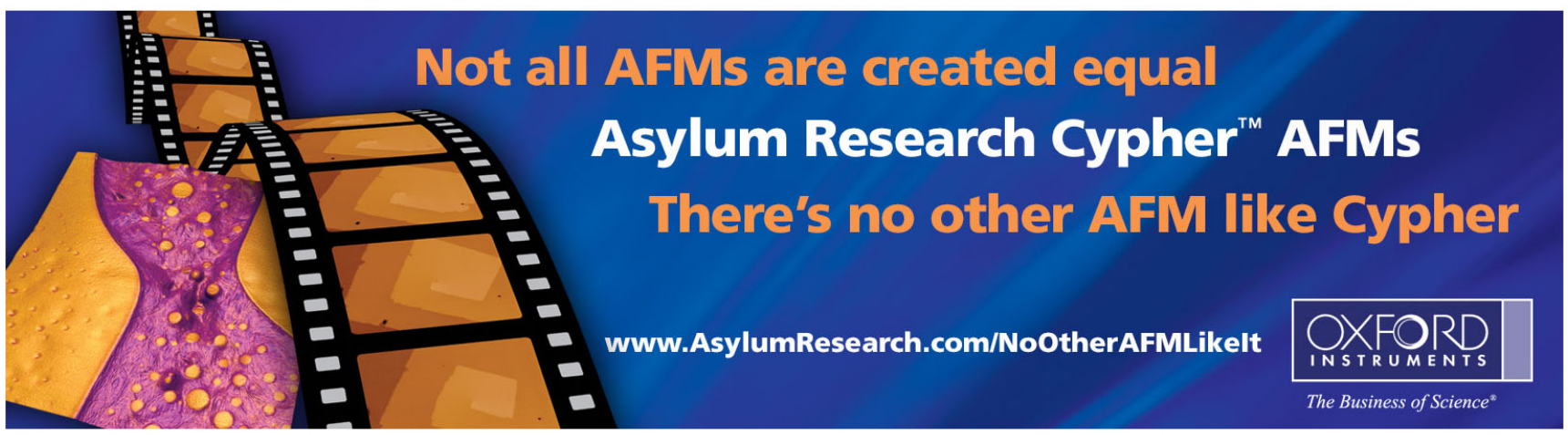




\title{
Exciton and multiexciton optical properties of single InAs/GaAs site-controlled quantum dots
}

\author{
J. Canet-Ferrer, ${ }^{1, a)}$ G. Munoz-Matutano, ${ }^{1}$ J. Herranz, ${ }^{2}$ D. Rivas, ${ }^{1}$ B. Alen, ${ }^{2}$ Y. Gonzalez, ${ }^{2}$ \\ D. Fuster, ${ }^{2}$ L. Gonzalez, ${ }^{2}$ and J. Martínez-Pastor ${ }^{1}$ \\ ${ }^{1}$ Institut de Ciència dels Materials, Universitat de València, P.O. Box 22085, 46071 Valencia, Spain

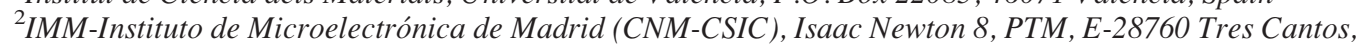 \\ Madrid, Spain.
}

(Received 4 September 2013; accepted 17 October 2013; published online 31 October 2013)

\begin{abstract}
We have studied the optical properties of InAs site-controlled quantum dots (SCQDs) grown on prepatterned GaAs substrates. Since InAs nucleates preferentially on the lithography motifs, the location of the resulting QDs is determined by the pattern, which is fabricated by local oxidation nanolithography. Optical characterization has been performed on such SCQDs to study the fundamental and excited states. At the ground state different exciton complex transitions of about $500 \mu \mathrm{eV}$ linewidth have been identified and the fine structure splitting of the neutral exciton has been determined $(\approx 65 \mu \mathrm{eV})$. The observed electronic structure covers the demands of future quantum information technologies. (C) 2013 AIP Publishing LLC. [http://dx.doi.org/10.1063/1.4828352]
\end{abstract}

One of the main interests in the quantum dot (QD) research is explained by their discrete electron density of states, optically revealed as a set of fundamental and excited transitions in the photoluminescence (PL) experiments. ${ }^{1-4}$ The carrier confinement in the QDs allows the formation of single charged, neutral exciton and multi-exciton complexes, which can be characterized by micro-photoluminescence ( $\mu$-PL). The electronic and optical properties of such QDs are sensitively modified by the exact number of charge carriers and their multiple spin configurations, QD shape, and stress or the doping surroundings, among others. ${ }^{5,6}$ Entangled photon pair sources, as required for quantum cryptography purposes, are found in radiative cascades from biexcitons to neutral excitons, ${ }^{7}$ while the spin manipulation, as required for quantum information memories, is more properly assisted by the trion transition (charged exciton). ${ }^{8}$ Coherent control of those phenomena has been recently achieved by manipulating charged and hot species (species with a carrier at the excited state). ${ }^{9}$ In charged biexcitons several decay pathways are observed, some of them involving different intermediate states in the cascade and presenting a high degree of polarization correlations. ${ }^{10}$ The use of hot trions enables the control of the spatial extent of carrier wavefunctions and the spin manipulation via optical pumping resonant to the P-Shell. ${ }^{11,12}$ From these findings, it is concluded that the detailed knowledge of the QD electronic structure is required for the generation and management of multiparticle exciton species with interesting emission properties for quantum information technologies.

The exciton complexes are commonly studied in low QD density samples using high spatial resolution optical microscopy. However, the use of deterministic positioned InAs QDs opens alternatives to design single quantum dot devices to fully exploit phenomena such as electron tunneling in quantum gates or strong coupling in cavity quantum electrodynamics. ${ }^{13-16} \mathrm{~A}$ few years ago, it was demonstrated that InAs QDs grown on $\operatorname{GaAs}(001)$ substrates patterned by means of lithography

a)Jose.Canet-Ferrer@uv.es techniques behave as single photon emitters. ${ }^{17}$ Since that moment, a great effort has been devoted to optimize the optical properties of site-controlled QDs (SCQDs). ${ }^{18}$ Nowadays, the wafer can be patterned by different techniques such as e-beam, focused ion beam, or atomic force microscopy (AFM), among other lithography methods. From these methods we focus on the AFM local oxidation nanolithography, since it reduces the number of patterning and cleaning processing steps in comparison to the other lithography techniques. In addition, this technique can be carried out in delicate samples maintaining the high resolution alignment capabilities and allowing epitaxial re-growth, as demonstrated by patterning directly on existing photonic crystal membranes. ${ }^{19}$

In the present work, the optical properties of this kind of SCQDs are examined to determine their technological possibilities. We find a characteristic electronic structure formed by a ground state and two different excited states, one of them nominally forbidden. At the ground state, different exciton complexes are identified by $\mu$-PL experiments. The fine structure splitting (FSS) of the neutral exciton is measured by means of polarization resolved $\mu$-PL measurements. Although the homogeneous linewidth is rather large, these QDs fulfill some basic demands mostly when an accurate control of the emitter position is required, as for example photonic devices based on the spatial tuning of Purcell effect. ${ }^{14,20}$ Finally, we have measured the energy splitting of the excited states to be compared with the neutral exciton energy. The existence of a level structure with well-defined P-states and the possibility of charge injection through the nominally forbidden transition promise a better control of the optical feeding, polarization and spin manipulation in our SCQDs.

The samples were grown on epitaxial GaAs(001) substrates, consisting of $500 \mathrm{~nm}$ thick GaAs layers grown on epi-ready commercial substrates by MBE. These GaAs epitaxial substrates are patterned by AFM local oxidation nanolithography using a commercial Nanotec AFM system under ambient conditions in tapping mode. The pattern consists of square arrays of $10 \mathrm{~nm}$ average high and $160 \mathrm{~nm}$ 
average wide oxide motifs with a periodicity of $2 \mu \mathrm{m}$. The MBE regrowth process on patterned substrates starts with in situ oxide desorption by atomic $\mathrm{H}$ exposure of the GaAs surface at $\mathrm{T}_{\mathrm{s}}=490^{\circ} \mathrm{C}$ for $30 \mathrm{~min}$ while $\mathrm{As}_{4}$ is also supplied in order to avoid As losses. This process efficiently removes the patterned oxide motifs, leading to the formation of nanoholes at the predefined positions while providing a clean surface for the growth of nanostructures with good optical properties. ${ }^{17}$ Prior to InAs deposition, a $15 \mathrm{~nm}$ thick GaAs buffer layer is grown at low temperature $\left(\mathrm{T}_{\mathrm{S}}=490^{\circ} \mathrm{C}\right)$ in order to preserve the pattern motifs. On top of the GaAs buffer layer, 1.5 monolayers (ML) of InAs are deposited at a growth rate of $0.01 \mathrm{ML} / \mathrm{s}$ at $\mathrm{T}_{\mathrm{S}}=490^{\circ} \mathrm{C}$. This InAs coverage is below the critical thickness for QD formation as observed by 2D-3D RHEED transition on non-patterned substrates (1.7 ML) under these growth conditions, but it is enough for obtaining nucleation of InAs inside the nanoholes to fabricate InAs SCQD. The InAs are distributed forming shallow nanostructures about $7 \mathrm{~nm}$ height and $90 \mathrm{~nm}$ width, as concluded from AFM characterization. ${ }^{17}$ This reduced aspect ratio (height to width) suggests disk- or lens-like nanostructures. After $2 \mathrm{~min}$ of growth interruption under $\mathrm{As}_{4}$ flux, samples are capped with $20 \mathrm{~nm}$ thick GaAs layer for optical characterization. The positioning technique could be limited by two main factors, on the one side, due to the non-linear response of the AFM scanner, and, on the other side, because of the size of the nanohole after the oxide removal. In our system, the resolution is essentially limited by the nanohole size since the scanner motion is corrected by software using an accurate calibration method, and by hardware using a close-phase loop circuit. In these conditions, we can estimate a lateral resolution of the positioning of below $50 \mathrm{~nm}$ (i.e., $1 / 4$ nanohole width) because InAs clearly nucleates at the bottom of the nanohole. The optical properties of the SCQDs were studied by confocal $\mu \mathrm{PL}$, using a diffraction limited fiber based confocal setup $(\mathrm{NA}=0.6)$ working at the liquid helium temperature. The detection of the $\mu$-PL signal was performed by means of a silicon back thinned CCD attached to a $0.3 \mathrm{~m}$ focal length double-spectrometer. A Silicon Avalanche Photodetector connected to a Time Correlated Single Photon Counting electronics was used for time resolved $\mu$-PL acquisition while a Ti: Sapphire laser under continuous wave or fs pulsed operation at $780 \mathrm{~nm}$ was used as excitation source.

Figure 1(a) consists of an AFM image of a rectangular array of GaAs oxide motifs with a pitch period of $2 \mu \mathrm{m}$. After the etching and the subsequent growth, the lithography motifs are replaced by InAs SCQDs while InAs nucleation in the non-patterned region is completely suppressed. A $\mu$-PL image of the same area is shown in Fig. 1(b) to show the high selectivity of the InAs deposition. This image is obtained by integrating multiple spectra in a $60 \mathrm{~nm}$ wide spectral window around $985 \mathrm{~nm}(\approx 1.22-1.30 \mathrm{eV})$. Some SCQDs emit either at the edges or out of the detection window, as corroborated by acquiring spectra at the corresponding wavelengths. Moreover, the periodicity found from the $\mu$-PL image perfect matches the nominal value $(2 \mu \mathrm{m})$ [Fig. 1(b)].

Figure 2(a) shows a series of $\mu-\mathrm{PL}$ spectra measured by increasing the excitation power in a representative SCQD from the array. Several peaks are observed around $1.24 \mathrm{eV}$
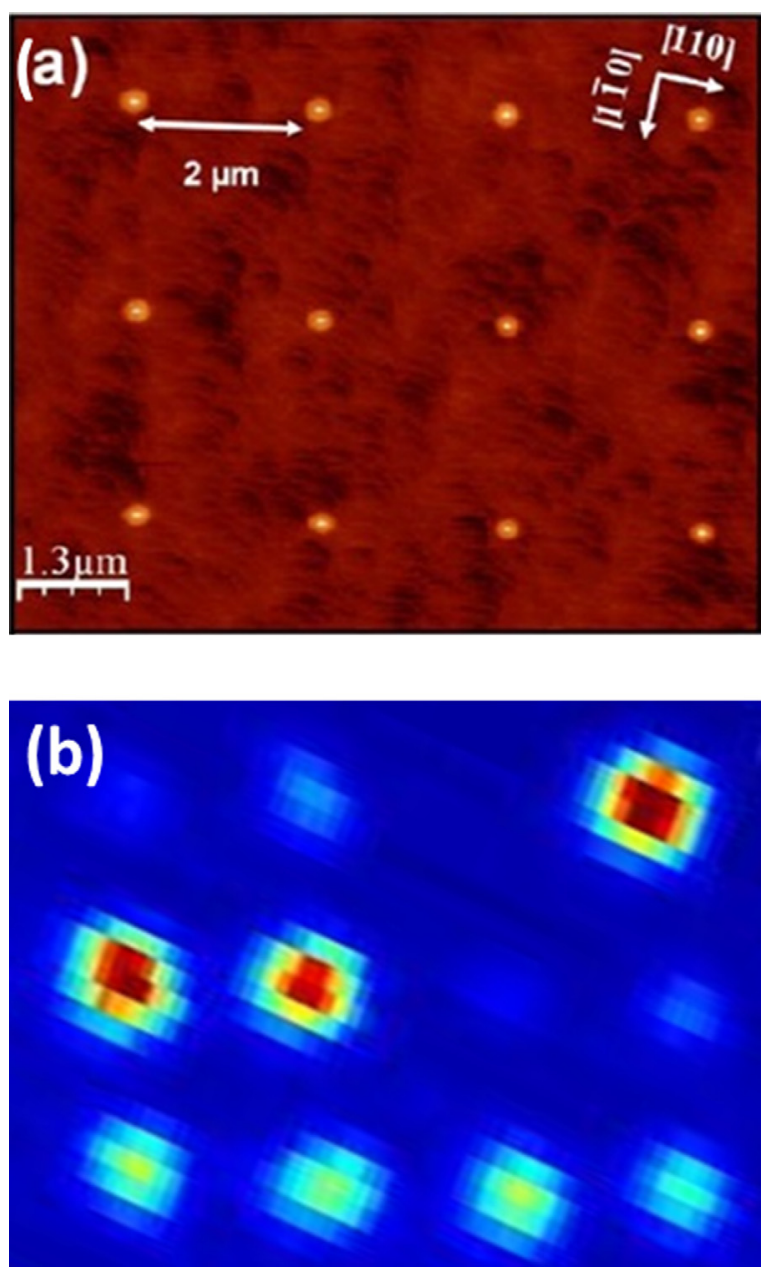

FIG. 1. (a) AFM image in a region of the $7 \times 7 \mathrm{GaAs}$ oxide matrix obtained by local oxidation atomic force microscopy lithography. (b) $\mu$-PL image of the same area after fabrication of the site controlled QDs.

presenting linewidths in the order of $500 \mu \mathrm{eV}(200-700 \mu \mathrm{eV}$ depending on the particular transition) and lifetimes close to $1.1 \mathrm{~ns}$ for the exciton-like lines and $0.56 \mathrm{~ns}$ in the case of the biexciton [see $\mu \mathrm{PL}$ transients in Fig. 2(b)]. Wang et al. demonstrated an important broadening of the PL lines in InAs/GaAs self-assembled QD when the wetting-layersurface distance is reduced below $40 \mathrm{~nm}$, accompanied by a strong reduction of the emission efficiency and recombination times for distances below $20 \mathrm{~nm} .^{21}$ Such broadening can be explained by the spectral diffusion effects since random carrier trapping to the QD surrounding defects or impurity vacancies induces electric field fluctuations with time, originating a slow stochastic movement of the peak energy. ${ }^{22}$ In our SCQDs the thickness of both GaAs capping and buffer layer are thin, 20 and $15 \mathrm{~nm}$, respectively, and residual defects can be expected arising from the cleaning process and crystal quality (impurities, roughness, point defects, etc.), arising from the atomic $\mathrm{H}$ oxide removal step performed just before GaAs buffer layer epitaxial growth. In spite of this a priori consideration, the exciton recombination times measured in our SCQDs are not different from those typically measured in self-assembled QDs and hence non radiative recombination paths are not present, even if we have a noticeable spectral diffusion broadening. 

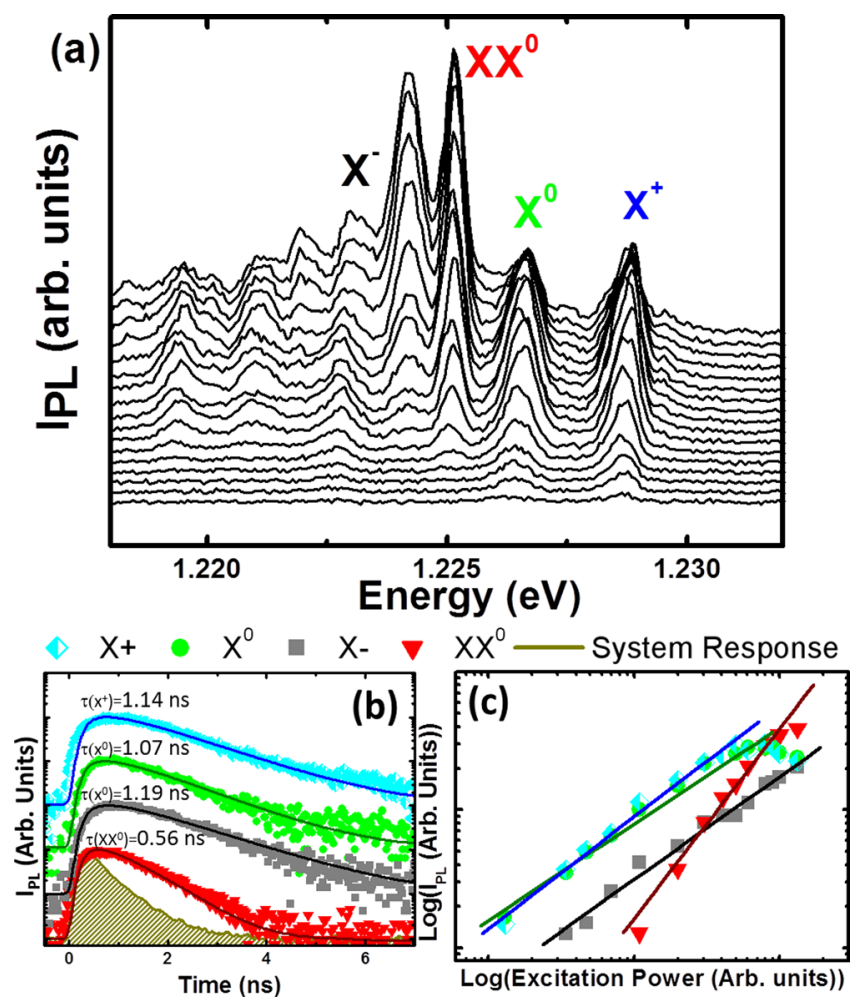

FIG. 2. (a) $\mu$-PL spectra for different excitation powers in a representative SCQD, (b) $\mu$-PL transients, and (c) logarithmic plot of the $\mu$-PL integrated intensity versus excitation power for optical transitions identified in (a).

In Fig. 2(c) the biexciton line is distinguished from the exciton-like ones by their superlinear dependence on the excitation power. The nature of the other optical transitions can be labeled taking into account the information from their spectra, as their binding energies, integrated intensity power dependence, polarization dependence and recombination times. The most usual spectral signatures of InAs/GaAs selfassembled QDs emitting around 1.2-1.3 eV are: (i) the emission energy of the negatively charged trion $\left(\mathrm{X}^{-}\right)$is observed typically $4 \mathrm{meV}$ below that of the neutral exciton emission (bonding position); ${ }^{23}$ (ii) the emission energy of the positively charged trion $\left(\mathrm{X}^{+}\right)$is above the neutral exciton (anti-bonding position); ${ }^{24,25}$ (iii) the emission energy of the biexciton $\left(\mathrm{XX}^{0}\right)$ can be either bonding or anti-bonding, but typically around $1 \mathrm{meV}$ from the neutral exciton $\left(\mathrm{X}^{0}\right)$ line. ${ }^{24-26}$ The $\mu$-PL spectra of our SCQDs fit with the energy splitting of exciton and multiexciton species described above. Moreover, the initial labeling in Fig. 2(a) is in concordance with the slopes found at the double logarithmic plot of the integrated intensity in Fig. 2(c). The neutral exciton presents the smallest slope, $\mathrm{m}\left(\mathrm{X}^{0}\right)=0.7$, while the slope of the biexciton is a factor two larger, $\mathrm{m}\left(\mathrm{XX}^{0}\right)=1.38$. $^{27,28}$ The identification of $\mathrm{XX}^{0}$ does not present any doubt, because in addition its decay time is half of that measured for the exciton-like species [Fig. 2(b)]. In the case of the $\mathrm{X}^{-}$ and $\mathrm{X}^{+}$trions the intensity-vs-power slopes $(0.75$ and 0.8 , respectively) are close to that of the neutral exciton, as expected from the statistical process of carrier capture. ${ }^{29}$

The FSS obtained from the polarization resolved $\mu$-PL spectra in Fig. 3 also confirms our previous assignment for $\mathrm{X}^{0}$ and $\mathrm{XX}^{0}$, given that their peaks experience opposite shifts upon a $90^{\circ}$ turn of the linear polarization analyzer with respect
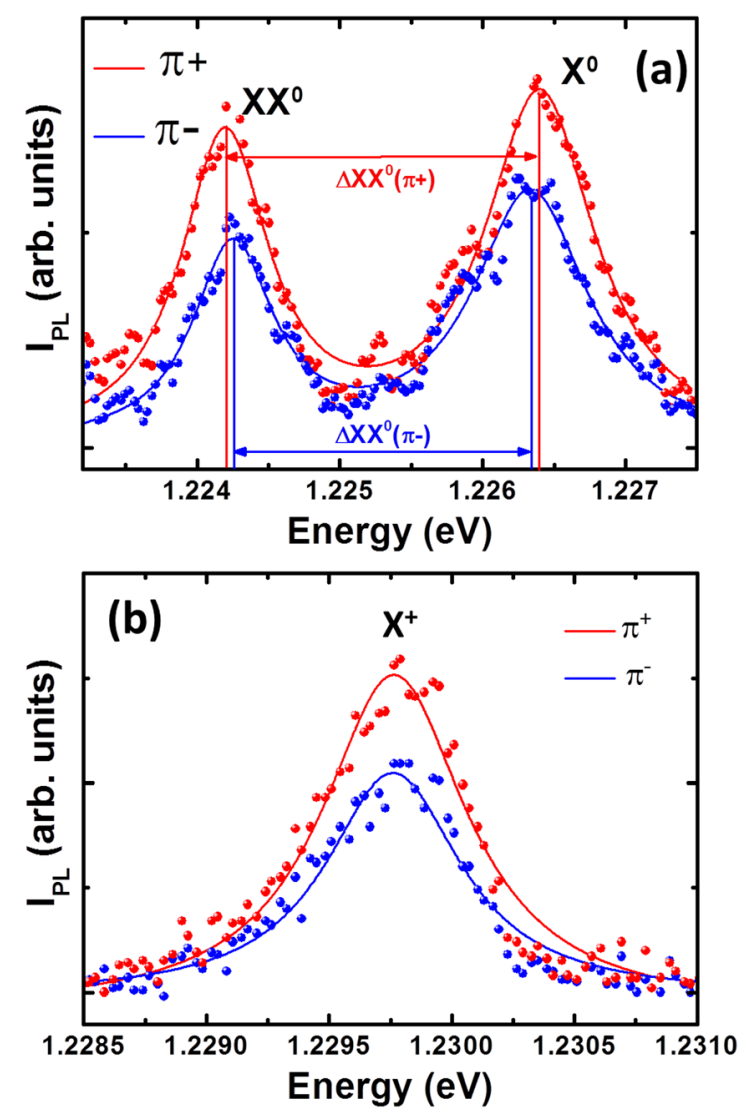

FIG. 3. Polarization resolved $\mu-\mathrm{PL}$ of $\mathrm{X}^{0}-\mathrm{XX}^{0}$ (a) and $\mathrm{X}^{+}$(b) species in a representative SCQD.

to the ${ }^{1-10}$ crystal direction. Furthermore, any detectable shift is observed for $\mathrm{X}^{+}$(within the constraints imposed by the emission linewidths of these QDs). The measured FSS is $\approx 65 \mu \mathrm{eV}$, comparable to values measured in self-assembled QDs emitting at similar energies. ${ }^{30,31}$ The other peaks appearing in the $\mu$ PL spectra in Fig. 2(a) exhibit a superlinear behavior at bonding energy positions, as expected for negatively charged multiexciton species of self-assembled InAs/GaAs QDs. ${ }^{32,33}$ These peaks can be identified with more precise experiments, out of the scope of the present paper.

The binding energies found for the different spectral lines identified in several SCQDs of the same patterned area are listed in Table I (sorted by ascending neutral exciton emission energy). These binding energies agree with those reported for lens-shaped InAs/GaAs QDs, ${ }^{27,29}$ although their energy splitting dependence on the ground state energy presents certain differences. In comparison to self-assembled InAs/GaAs QDs, for which biexcitons change from bonding to anti-bonding energy position when increasing the $\mathrm{X}^{0}$ energy,

TABLE I. Energy splitting of the exciton complexes in several SCQDs.

\begin{tabular}{lccc}
\hline \hline$X^{0}(\mathrm{eV})$ & $X X^{0}-X^{0}(\mathrm{meV})$ & $X^{-}-X^{0}(\mathrm{meV})$ & $X^{+}-X^{0}(\mathrm{meV})$ \\
\hline 1.2129 & -2.6 & - & - \\
1.2228 & -2.1 & -5.9 & - \\
1.2264 & -1.3 & -3.3 & 2.2 \\
1.2339 & -1.8 & -3.5 & 1.5 \\
1.2610 & -2.3 & -4.4 & - \\
1.2697 & -2.5 & -4.7 & - \\
\hline \hline
\end{tabular}


(a)

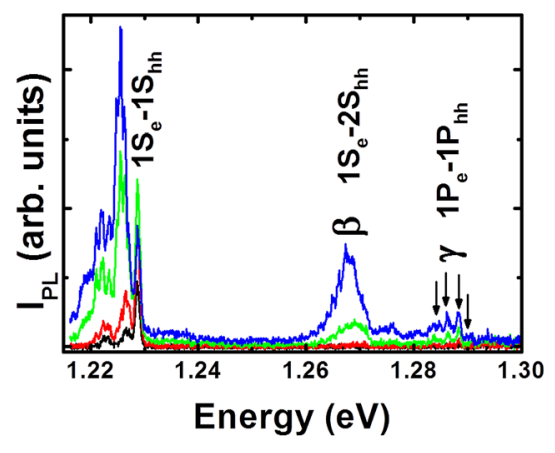

FIG. 4. (a) $\mu \mathrm{PL}$ spectra as a function of the excitation power and (b) scheme of the electronic structure and non-resonant optical injection channels. Notice that in the presence of a hole in the ground state optical injection via $\beta$ transition drives to the $2 \mathrm{~S}$-Shell hot trion, allowing recombination without phonon emission; in contrast if the electron-hole pair is injected through the $\gamma$ transition the electron must be thermalized to the ground state before recombination.

in all studied SCQDs the biexciton and negative trion exhibit negative binding energies larger than $\sim 1.3 \mathrm{meV}$ and $3.3 \mathrm{meV}$ respectively. This fact can be due to the particular confinement potential in our SCQDs exhibiting a different geometrical shape or composition (In-Ga mixing effect).

Figure 4 shows the excitation power evolution of the $\mu-\mathrm{PL}$ spectrum in a representative SCQD covering the energy range for the excited state emission. Two groups of optical transitions are labeled as $\beta$ and $\gamma$, appearing above $20 \mu \mathrm{W}$ of irradiated power. These transitions occurring around 40 and $55 \mathrm{meV}$ above the ground state optical transition, must be related to different excited states in our QDs. As occurs with the excited states of self-assembled QDs, the energy shift for $\beta$ and $\gamma$ transitions present certain dependence on the exciton confinement energy (see Table II), but we would need SCQDs emitting at lower energies to establish a better comparison. The $\beta$ transition is composed by a single peak at $1.268 \mathrm{eV}$, while in $\gamma$ we can distinguish several peaks at $1.284,1.286,1.289$, and $1.290 \mathrm{eV}$. The number of lines and their positions are consistent with those obtained

TABLE II. Energy splitting of the excited states in SCQDs emitting at different energies.

\begin{tabular}{lcc}
\hline \hline $1 \mathrm{~S}_{\mathrm{e}}-1 \mathrm{~S}_{\mathrm{hh}}(\mathrm{eV})$ & $1 \mathrm{~S}_{\mathrm{e}}-2 \mathrm{~S}_{\mathrm{hh}}(\mathrm{eV}) \Delta \mathrm{S}(\mathrm{meV})$ & $1 \mathrm{P}_{\mathrm{e}}-1 \mathrm{P}_{\mathrm{hh}}(\mathrm{eV}) \Delta \mathrm{P}(\mathrm{meV})$ \\
\hline 1.213 & 1.268 & 1.286 \\
& 39 & 57 \\
1.265 & 1.308 & 1.321 \\
& 43 & 56 \\
1.284 & 1.325 & - \\
& 41 & \\
1.300 & - & 1.341 \\
& 41 & 1.369 \\
1.315 & 1.336 & 54 \\
& 21 & 1.357 \\
1.321 & 1.337 & 36 \\
& 16 & 1.411 \\
1.349 & 1.398 & 62 \\
& 48 & \\
\hline \hline
\end{tabular}

by atomistic calculations in lens-shaped $\operatorname{In}(\mathrm{Ga})$ As QDs made by Narvaez and Zunger. ${ }^{26}$ The $\gamma$-multiplet structure has been previously measured on InAs self-assembled QDs, ${ }^{34}$ while the $\beta$ transition is attributed to a nominally forbidden transition among unpaired shells, which becomes allowed because of the finite band offsets and orbital mixing effects. We tentatively describe the optical emission of our SCQDs as depicted in Fig. 4(b). As an advantage with respect to typical self-assembled QDs, the strength of nominally forbidden transitions $(\beta)$ will allow a better control of the spin dynamics since it drives to an additional charge injection and polarization control possibilities, e.g., injection of $1 \mathrm{~S}$ electrons via the $1 \mathrm{~S}_{\mathrm{e}}-2 \mathrm{~S}_{\mathrm{hh}}$ channel (non-resonant to the ground state). At the same time, it enables the managing of trions and biexcitons with a spectator charge at the $2 \mathrm{~S}$-Shell, expanding the number of available excited species.

In summary, we have studied the emission spectra of site controlled InAs/GaAs QDs fabricated by local oxidation nanolithography and MBE re-growth. The exciton ground state emission lines of our SCQDs occurs in the range of $1.2-1.3 \mathrm{eV}$ and are characterized by rather large average linewidths of around $500 \mu \mathrm{eV}$ in average with a FSS of about $65 \mu \mathrm{eV}$. Different exciton complexes have been identified from their measured binding energy, PL power dependence, radiative recombination times, and polarization resolved $\mu \mathrm{PL}$ spectra. The identification of exciton especies is consistent with the data found in literature for lens shape InAs Self-Assembled quantum dots, although we found important dispersion in the binding energy dependence on the ground state energy, for the exciton complexes and for their excited states. Compositional and/or geometrical changes might be the origin for such dispersion. The optical properties of our SCQDs fit very well with the new trends in optoelectronics, mainly for those devices where an accurate control of the emitter location is mandatory. Moreover, the electronic structure found in these QDs results promising for further applications since an additional optical injection channel is enabled via excited state pumping. The understanding and control of these properties is of paramount importance for the incoming quantum information technologies.

The authors want to acknowledge the financial support from Spanish government through grants QD-NANOTICS: TEC2011-29120-C05-01, EPIC-NANOTICS: TEC201129120-C05-04, PROMESA: ENE2012-37804-C02-02, and Q\&C Light: S2009ESP-1503. We also thank the support from the PROMETEO2009/74 project (Generalitat Valenciana). J.C.-F. thanks the Spanish MCI for his FPI grant (No. BES-2006-12300) and J.H. acknowledges to the JAE program for the funds.

\footnotetext{
${ }^{1}$ D. Bimberg, Semiconductor Nanostructures (Springer, Berlin, 2008).

${ }^{2}$ C. Santori, D. Fattal, J. Vuckovic, G. S. Solomon, and Y. Yamamoto, Nature 419, 594 (2002).

${ }^{3}$ V. Zwiller, H. Blom, P. Jonsson, N. Panev, S. Jeppesen, T. Tsegaye, E. Goobar, M.-E. Pistol, L. Samuelson, and G. Björk, Appl. Phys. Lett. 78, 2476 (2001).

${ }^{4}$ M. A. Pooley, D. J. P. Ellis, R. B. Patel, A. J. Bennett, K. H. A. Chan, I. Farrer, D. A. Ritchie, and A. J. Shields, Appl. Phys. Let. 100, 211103 (2012).

${ }^{5}$ M. Ediger, G. Bester, A. Badolato, P. M. Petroff, K. Karrai, A. Zunger,
} and R. J. Warburton, Nat. Phys. 3, 774 (2007). 
${ }^{6}$ Y. Benny, Y. Kodriano, E. Poem, D. Gershoni, T. A. Truong, and P. M. Petroff, Phys. Rev. B 86, 085306 (2012).

${ }^{7}$ N. Akopian, N. H. Lindner, E. Poem, Y. Berlatzky, J. Avron, D. Gershoni, B. D. Gerardot, and P. M. Petroff, Phys. Rev. Lett. 96, 130501 (2006).

${ }^{8}$ T. M. Godden, J. H. Quilter, A. J. Ramsay, Y. Wu, P. Brereton, S. J. Boyle, I. J. Luxmoore, J. Puebla-Nunez, A. M. Fox, and M. S. Skolnick, Phys. Rev. Lett. 108, 017402 (2012).

${ }^{9}$ Y. Kodriano, I. Schwartz, E. Poem, Y. Benny, R. Presman, T. A. Truong, P. M. Petroff, and D. Gershoni, Phys. Rev. B 85, 241304(R) (2012); K. Muller, T. Kaldewey, R. Ripszam, J. S. Wildmann, A. Bechtold, M. Bichler, G. Koblmuller, G. Abstreiter, and J. J. Finley, Sci. Rep. 3, 1906 (2013).

${ }^{10}$ E. Poem, Y. Kodriano, C. Tradonsky, B. D. Gerardot, P. M. Petroff, and D. Gershoni, Phys. Rev. B 81, 085306 (2010).

${ }^{11}$ A. B. Henriques, A. Schwan, S. Varwig, A. D. B. Maia, A. A. Quivy, D. R. Yakovlev, and M. Bayer, Phys. Rev. B 86, 115333 (2012).

${ }^{12}$ S. G. Carter, Ş. C. Bădescu, and A. S. Bracker, Phys. Rev. B 81, 045305 (2010); G. Muñoz-Matutano, M. Royo, J. I. Climente, J. Canet-Ferrer, D. Fuster, P. Alonso-González, I. Fernández-Martínez, J. Martínez-Pastor, Y. González, L. González, F. Briones, and B. Alén, ibid. 84, 041308(R) (2011).

${ }^{13}$ P. W. Li, W. M. Liao, D. M. T. Kuo, S. W. Lin, P. S. Chen, S. C. Lu, and M.-J. Tsai, Appl. Phys. Lett. 85, 1532 (2004).

${ }^{14}$ K. Hennessy, A. Badolato, M. Winger, D. Gerace, M. Atatüre, S. Gulde, S. Fält, E. L. Hu, and A. Imamoglu, Nature 445, 896 (2007).

${ }^{15}$ T. Ishikawa, T. Nishimura, S. Kohmoto, and K. Asakawa, Appl. Phys. Lett. 76, 167-169 (2000); I. Sychugov, Y. Nakayama, and K. Mitsuishi, Nanotechnology 21, 285307 (2010); J. Y. Lee, M. J. Noordhoek, P. Smereka, H. Mc Kay, and J. M. Millunchick, ibid. 20, 285305 (2009); A. Pierret, M. Hocevar, S. L. Diedenhofen, R. E. Algra, E. Vlieg, E. C. Timmering, M. A. Verschuuren, G. W. G. Immink, M. A. Verheijen, and E. Bakkers, ibid. 21, 065305 (2010); K. H. Chen, C.-Y. Chien, and P.-W. Li, ibid. 21, 055302 (2010); A. Bernardi, M. I. Alonso, A. R. Goñi, J. O. Osso, and M. Garriga, Surf. Sci. 601, 2783-2786 (2007); B. Bruhn, F. Sangghaleh, and J. Linnros, Phys. Status Solidi A 208, 631 (2011); P. Atkinson, O. G. Schmidt, S. P. Bremner, and D. A. Ritchie, C. R. Phys. 9, 788 (2008).

${ }^{16}$ J. Martin-Sanchez, P. Alonso-Gonzalez, J. Herranz, Y. González, and L. Gonzalez, Nanotechnology 20, 125302 (2009); J. Martin-Sanchez, Y. González, P. Alonso-Gonzalez, and L. Gonzalez, J. Cryst. Growth 310, 4676-4680 (2008).

${ }^{17}$ J. Martín-Sánchez, G. Munoz-Matutano, J. Herranz, J. Canet-Ferrer, B. Alen, Y. Gonzalez, P. Alonso-Gonzalez, D. Fuster, L. Gonzalez, J. Martinez-Pastor, and F. Briones, ACS Nano 3, 1513 (2009).

${ }^{18}$ J. Tommilla, A. Schramm, T. V. Hakkarainen, M. Dumitrescu, and M. Guina, Nanotechnology 24, 235204 (2013); C. Schneider, A. Huggenberger,
M. Gschrey, P. Gold, S. Rodt, A. Forchel, S. Reitzenstein, S. Höfling, and M. Kampet, Phys. Status Solidi A 209, 2379 (2012); J. Lee, T. W. Saucer, A. J. Martin, D. Tien, J. M. Millunchick, and V. Sih, J. Lumin. 133, 117 (2013).

${ }^{19}$ I. Prieto, J. Herranz, Y. González, P. A. Postigo, B. Alén, L. González, J. Martín-Sánchez, L. J. Martínez, M. Kaldirim, D. Fuster, J. Canet-Ferrer, G. Muñoz-Matutano, and J. Martínez-Pastor, 13th International Conference on Transparent Optical Networks (ICTON), Sweden Jun 26-30, (2011).

${ }^{20}$ J. Canet-Ferrer, L. J. Martínez, I. Prieto, B. Alén, G. Muñoz-Matutano, D. Fuster, Y. González, M. L. Dotor, L. González, P. A. Postigo, and J. Martínez-Pastor, Opt. Express 20, 7901 (2012).

${ }^{21}$ C. F. Wang, A. Badolato, I. Wilson-Rae, P. M. Petroff, E. Hu, J. Uruyama, and A. Imamoglu, Appl. Phys. Lett. 85, 3423 (2004).

${ }^{22}$ H. Kamada and T. Kutsuwa, Phys. Rev. B 78, 155324 (2008); P. AlonsoGonzalez, B. Alen, D. Fuster, Y. Gonzalez, L. Gonzalez, and J. MartínezPastor, Appl. Phys. Lett. 91, 163104 (2007).

${ }^{23}$ R. J. Warburton, C. Schäfen, D. Haft, F. Bickel, A. Lorke, K. Karrai, J. M. Garcia, W. Schoenfeld, and P. M. Petroff, Nature 405, 926 (2000).

${ }^{24}$ S. Rodt, A. Schliwa, K. Pötschke, F. Guffarth, and D. Bimberg, Phys. Rev. B 71, 155325 (2005).

${ }^{25}$ A. Schliwa, M. Winkelnkemper, and D. Bimberg, Phys. Rev. B 79, 075443 (2009).

${ }^{26}$ G. A. Narvaez, G. Bester, and A. Zunger, Phys. Rev. B 72, 245318 (2005).

${ }^{27}$ M. Abbarchi, C. A. Mastrandrea, T. Kuroda, T. Mano, K. Sakoda, N. Koguchi, S. Sanguinetti, A. Vinattieri, and M. Gurioli, Phys. Rev. B 78, 125321 (2008).

${ }^{28}$ L. Seravalli, G. Trevisi, P. Frigeri, D. Rivas, G. Muñoz-Matutano, I. Suarez, B. Alen, J. Canet-Ferrer, and J. Martinez-Pastor, Appl. Phys. Lett. 98, 173112 (2011).

${ }^{29}$ M. Nakayama, K. Suyama, and H. Nishimura, Phys. Rev. B 51, 7870 (1995).

${ }^{30}$ R. Seguin, A. Schliwa, S. Rodt, K. Pötschke, U. W. Pohl, and D. Bimberg, Phys. Rev. Lett. 95, 257402 (2005).

${ }^{31}$ W. Langbein, P. Borri, U. Woggon, V. Stavarache, D. Reuter, and A. D. Wieck, Phys. Rev. B 69, 161301(R) (2004).

${ }^{32}$ E. Poem, J. Shemesh, I. Marderfeld, D. Galushko, N. Akopian, D. Gershoni, B. D. Gerardot, A. Badolato, and P. M. Petroff, Phys. Rev. B 76, 235304 (2007).

${ }^{33}$ B. Urbaszek, E. J. McGhee, J. M. Smith, R. J. Warburton, K. Karrai, B. D. Gerardot, J. M. Garcia, and P. M. Petroff, Phys. E 17, 35 (2003).

${ }^{34}$ G. Munoz-Matutano, B. Alen, J. Martinez-Pastor, L. Seravalli, P. Frigeri, and S. Franchi, Nanotechnology 19, 145711 (2008). 